Please cite this chapter as: Jennett, C., Cognetti, E., Summerfield, J. and Haklay, M. 2016. Usability and interaction dimensions of participatory noise and ecological monitoring. In Loreto, V., Haklay, M., Hotho, A., Servedio, V.C.P, Stumme, G., Theunis, J., Tria, F. (eds.) Participatory Sensing, Opinions and Collective Awareness. Springer. pp.201-212.

\title{
Usability and interaction dimensions of participatory noise and ecological monitoring
}

\author{
Charlene Jennett, Eleonora Cognetti, Joanne Summerfield, Muki Haklay \\ UCL Interaction Centre \\ University College London (UCL) \\ Gower Street, London WC1E 6BT, \\ United Kingdom
}

\section{Introduction}

The integration of sensors in smartphones has transformed personal mobile phones, from tools primarily meant for communication purposes, into instruments that can sense or collect information about the surrounding environment (Lane et al., 2010). There are several mobile apps that allow ordinary members of the public (non-professionals) to collect fine-grained data about their environment and to contribute to real research. By involving citizens in environmental monitoring activities this helps to raise their awareness of environmental issues (Becker et al., 2013). However there are also challenges in the use of such apps. The lower-end sensors for mobile phones do not give the same level of data accuracy as specialised devices. The data is being collected by 'inexpert' citizens, which may add to perceptions of the data being inaccurate. Also, in order to apply complex statistical models, the data needs to be collected in mass quantities. This raises two questions about the citizens involved: (1) what is their experience of using the app and (2) what factors motivate them to participate?

In this chapter we describe field studies where we tested two kinds of environmental monitoring apps - noise monitoring apps (WideNoise, NoiseWatch) and ecological monitoring apps (iSpot, Project Noah, UK Ladybird Survey). These studies were conducted with the aim of uncovering factors that acted as barriers to data collection and to identify design opportunities that could sustain user contribution. It was important for us to conduct our studies 'in the wild' (outside of the lab) as we wanted to understand the factors that affect how a person uses a technology within a natural context (Rogers, 2011). For both studies, participants tried out several apps in various locations around London. For the noise monitoring 
study, participants additionally took part in a 6-day diary study, where they reflected on their experience of using (and not using) the apps during their day-today lives. In the following sections we will describe the aim, methodology and findings of each of our studies. This will be followed by a discussion of the lessons learned.

\section{Noise Monitoring}

Environmental noise is defined as "the noise cause by traffic, industrial and recreational activities" (European Commission, 1996). It is estimated that approximately $20 \%$ of the EU population ( 80 million people) are exposed to daytime noise levels above $65 \mathrm{dBA}$ and that another 170 million people live in areas with noise levels between 55 and $65 \mathrm{dBA}$ (European Commission, 1996). As a result of urban noise pollution, approximately $25 \%$ of the EU population suffer from a deterioration of life quality due to annoyance, and between 5 to $15 \%$ are affected by sleep disturbance (European Parliament and Council, 2002). To tackle this problem, the EU issued the European Noise Directive. They aim to establish a noise management policy, which includes generating noise maps every 5 years to monitor the levels of noise generated by road, air and rail traffic, as well as industrial facilities. However this approach is limited because it does not provide a finegrained view of actual exposure to environmental noise; it is based on simulation models based on noise samples collected in limited areas (Stevens, 2012).

Advocates of participatory noise mapping argue that if enough citizens could be encouraged to go out and collect noise readings, then this could provide the more fine-grained level of data that is needed. There are currently several mobile phone apps for noise detection that have been developed and made available to download free of charge. In our study we decided to test two apps: NoiseWatch and WideNoise.

NoiseWatch is an app developed by the European Environment Agency. NoiseWatch presents a simple interaction focused on recording and submitting noise samples. A category selection list is provided and the user is tasked with classifying the noise heard: air traffic, road traffic, rail traffic, industrial activities, or 'other'.

WideNoise is an app developed by a consortium of academic and research institutions. WideNoise presents a more complex design than NoiseWatch, involving more steps for noise sampling and submission, but it also offers a more personalized experience. Users can guess the noise level while the measurement is being carried out, with the aim of teaching users to learn to identify the $\mathrm{dB}$ level of a noise. Users have the option of providing subjective feedback on their personal perception of the noise (e.g. love/hate, calm/hectic, social/alone). Users can add tags to express sentiment, or to provide more details about the place or noise source. It is also possible for users to create an online account where their personal 
contributions are displayed on a map and there are several graphs that show their contribution history and other data.

However not much is yet known about the experiences of the people that use these apps. Furthermore, previous research has focused on special interest groups that are highly motivated to participate - for example, members of a non-profit environmental organisation (D'Hont et al., 2012) or residents that lived near Heathrow airport (Becker et al., 2013. We suggest that it is important to sustain a wide pool of citizens' contributions in order for the participatory noise paradigm to be effective. Therefore in our research we decided to recruit participants that only had a minimal/moderate interest in noise issues. We investigated participants' experiences of two apps (NoiseWatch, WideNoise) with the aim of uncovering factors that acted as enablers and barriers to data collection, and identifying design opportunities that could encourage contribution.

\section{Methodology}

Initially we distributed a survey to 60 residents of London and surrounding areas, to gauge their interest in noise and environmental issues. These participants were recruited via an opportunity sample. Based on our survey data, we identified three types of contributors that could be good candidates for participatory noise mapping:

1. People concerned about noise effects on health, driven by personal interest;

2. People sensitive to noise, driven by personal interest and contribution purposes;

3. People already manifesting a pro-environment behaviour, driven by contribution purposes.

The survey respondents that most fit these criteria were invited to take part in our field study. We recruited 18 participants in total, 4 male and 14 female. Their ages ranged from 21 to 60 years (mode age category $=21-29$ years). Regarding occupation, 9 were professionals and 9 were students.

The 18 participants were instructed to install two noise monitoring apps NoiseWatch and WideNoise - on their personal smartphones. Seven participants installed the apps on their iPhone. Four participants installed the apps on Android smartphones. The seven remaining participants owned other kinds of Smartphones (e.g. Windows, Blackberry, Nokia) that were not compatible with the app. These participants were given a Samsung handset with the apps already installed.

The first part of the study was a one-hour field experiment. Ten participants performed the first part of the study in a workshop held at a London university campus; this involved testing the app in the streets surrounding the university campus area. The other eight participants executed this part of the study by them- 
selves because they were unable to attend the workshop; this involved testing the app outdoors on streets close to where they lived or on their journey to/from work. The researcher instructed all participants to test each app for 30 minutes each. To counteract order effects, half of the participants tested NoiseWatch first, and half of the participants tested WideNoise first. Participants were instructed to walk around the campus (around nearby roads) and to voice record their observations (using the voice recording app on their phone). Participants were also given a checklist of items to act as a prompt. This list included items such as: context in which the measurement was carried out, the experience of taking samples on the street, things that they found pleasant/unpleasant, easy/difficult, clear/unclear, etc. After the one-hour field experiment, participants reconvened with the researcher for a brief discussion of their experience. All participants were rewarded with a small gift voucher (£5) for taking part.

The second part of the study was a week-long diary study. The 18 participants were instructed to use the two apps for three consecutive days each and to voice record their observations daily. Again the order of which app to use first was counterbalanced amongst participants. Participants were instructed to send their voice recordings to the researcher every 2 days and in the event of a delay, the researcher sent the participant a reminder. At the end of the week, participants took part in a brief interview (in person or via Skype). We also held a raffle draw, where 1 participant was selected at random to receive a $£ 50$ gift voucher.

Participants' voice recordings were transcribed and analysed using Thematic Analysis- a qualitative method for identifying, analysing and reporting patterns (themes) within data (Braun \& Clarke, 2006). Our themes include: user interface, sense making, technology, lifestyle and motivation.

\section{User Interface}

Participants desired a fast interaction, good user interface contrast, visible controls and a low number of steps for taking and submitting noise data. Being able to express subjective perceptions of noise was also a desirable feature, and as a result the majority of participants preferred WideNoise over NoiseWatch. But at the same time, WideNoise involved more steps for noise sampling and data submission, which could prove problematic when trying to collect data on-the-go:

"I just took a sample on a busier street, I kind of had to sit down and concentrate a bit more as I was using it in public. The other places I was before were much quieter and I could sit and take my time, so that was a little frustrating but I enjoyed the features, though I felt a bit rushed through them." [F27, WideNoise]

"In some places especially with this app it takes so long! So I can't just quickly sample it." [M28, WideNoise]

Other problems encountered by participants included screen glare in the sunlight, poor contrast, small labels and fiddly controls. For example, in Figure 1 we 
can see a participant using a sheet of paper in an attempt to shade the phone screen from the sun.

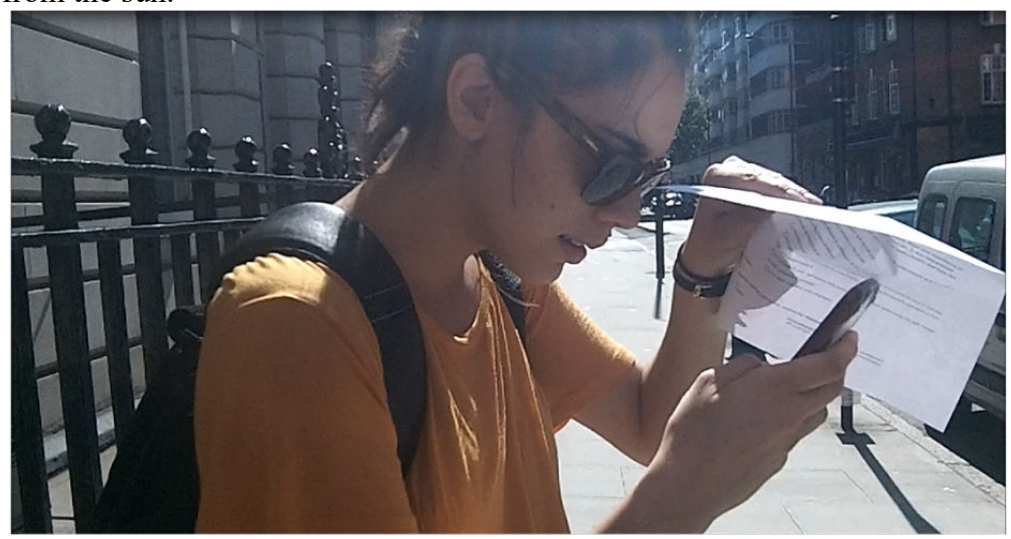

Figure 1. A participant using paper to overcome screen glare from the sun.

\section{Sense Making}

The use of $\mathrm{dB}$ numeric values was not particularly meaningful to participants. Several participants said that they would have liked to see a comparison with noise levels detected in other areas, or a history of their personal measurements. This would enable them to make inferences about their personal exposure to noise, as well as information about the effects on health of the exposure to those levels:

"I do not really know if $52 \mathrm{~dB}$ is good or bad. I just know it's a middle range noise. I guess it could give me advice for what side effects there are from being in this kind of level of noise for too long, I don't know, if there are side effects, I have no idea." [F27, NoiseWatch]

"I took a measurement when a car was passing by and interestingly the noise level went up to the very edge of good level of noise, 45, which is quite interesting, because this is the type of noise you are going to come across anyway in an urban environment with traffic, so obviously it's questionable whether it is good for your health to live anywhere near road traffic." [F33, NoiseWatch]

We suggest that a re-examination of how to best represent real world noises in the apps is needed. The current $\mathrm{dB}$ level categorization into 'good' and 'bad' is based on $\mathrm{dB}$ levels causing hearing damage. Typical $\mathrm{dB}$ levels in urban environments are lower; however they can still cause 'annoyance' and potentially affect a person's wellbeing over a longer period of time.

Another finding was that the classification of noises into a limited number of categories, as in NoiseWatch, clashed with the variety of noises people would perceive in the urban environment. Similarly the iconographic set of noise types pro- 
vided in WideNoise, automatically matching an icon to a noise recording, was perceived as unsuitable on several occasions:

"The main noise is coming from the air conditioning and WideNoise has identified air-conditioning as a TV which is quite amusing" [M36, WideNoise]

Icons are limited because they can be misinterpreted by users and it is difficult to indicate loudness and tonality at the same time.

\section{Technology}

Lack of internet connectivity can prevent sample collection and may discourage people from using the apps. Unlike WideNoise, NoiseWatch does not allow you to store samples and upload them at a later time when connection becomes available again or over a WiFi connection. For this reason, two participants did not use NoiseWatch during the week-long diary study because they were unwilling/ unable to consume their Internet data allowance.

Participants also expressed concerns about the GPS activation relative to battery consumption. In some cases participants decided to disable the GPS:

"I feel reluctant to open the GPS because it tends to consume the phone battery, so I chose not to use the GPS." [F34, both apps]

\section{Lifestyle and Motivation}

Lack of time, routine, and forgetfulness were identified as barriers for sustained contribution. Ten participants said that they had a routine lifestyle and once they collected noise samples along their daily routes they would not feel motivated to carry on sampling because of the lack of sense of discovery:

"So far, most of my samples have got the car and the feather icon, so after several measurements I find this becomes a bit boring, because I do not get the chance to guess others, because I do not go to places where they have higher or lower levels of noise. A bit of variation would be good." [F34, WideNoise]

Five participants talked about how they found themselves forgetting to take noise samples:

"I almost forgot to use it, and I kind of set up the alarm to remind me to use it. It's just very easy for people to forget to use it, well for me, I don't see too many opportunities to use it." [F28, both apps]

"The best time I have found I'd be able to take a noise sample would be when I am waiting, for example at the train station, that's the only place I find I am able to spend time to do so." [F34, both apps]

Finally, five participants listed amongst the reasons for not being likely to continue the activity the fact that they did not see how their contribution could make a 
difference. In line with previous research (Rotman et al., 2012), this suggests that there are different motivational factors affecting initial interest and sustained contribution. Users need compelling reasons to motivate them to continue taking noise readings over a long period of time.

\section{Ecological Monitoring}

The second type of activity that we explore here relates to ecological monitoring, specifically, monitoring within the field of biodiversity. In this context, the concept of 'biodiversity' - the number, variety and variability of organisms living in a certain area - plays a major role. Monitoring biodiversity is important because it allows conservationists to keep track of changes in a population. Involving members of the public in monitoring activities can help in raising awareness of ecological issues, scientific processes and the importance of conservation.

Contextual observation of two 'BioBlitz' events helped lay the foundation for the user evaluation. A BioBlitz is an event where members of the public are encouraged to participate in ecological citizen science. It takes place within a defined geographic area and over a 24 hour time period. They are organised as a series of walks or activities, for example bird walks, fungi forays, stream dipping, and butterfly walks. These events, led by subject matter experts, local and familiar with the ecology of the area, offer an opportunity for non-experts to gain experience of species identification and data collection.

We observed two BioBlitzes in the UK - one in Gloucestershire and one in Surrey organized by Natural England and Sutton Ecology Centre respectively. It was commonplace for experts to use paper-based forms to mark down their sightings and these forms were then handed to a data entry team. By contrast, novices took on a more passive role and did not collect any data themselves. We suggest that mobile technology could provide support for data collection to novices in the field. Firstly, it enables users to collect and submit data immediately rather than finding time post event to submit. Secondly, if designed appropriately, mobile technology could provide support for novices with species identification during data collection.

There are currently several mobile phone apps for ecological monitoring that are available to download. In our study we decided to test three apps: Project Noah, iSpot and UK Ladybird Survey. As was the case in noise monitoring, not much is yet known about the experiences of the people that use these apps, particularly the experiences of novice users. In our research we investigated participants' experiences of using these apps, exploring whether such apps supported novice users to engage in biodiversity monitoring. 


\section{Methodology}

Twenty-three participants were recruited via an opportunity sample. Seventeen were female, six were male, and their ages ranged from 24 and 64 years (mean = $42.9, \mathrm{SD}=13.5$ ). They were all novices, as they had never taken part in ecological monitoring before. They were tested in ten groups and the groups met at Hampstead Heath or Richmond Park. These are large open heathland areas, covering 790 acres and 2,360 acres respectively. Weather conditions ranged from very hot bright weather to thunderstorms. The researcher explained the purpose of the field study and gave each participant a Samsung Galaxy XCover mobile phone, where the apps were already installed. The order in which the apps were tested was counterbalanced across the 10 groups in order to counteract any order effects.

All three apps had distinctive approaches to the process of engaging users in data collection process. There were two multi-species apps: Project Noah, a USdeveloped app; and iSpot, an app developed by the Open University in the UK. To successfully complete a record, the user needs to take a photo, move through a series of data fields and tabs, locate the sighting and then submit to the 'community' for expert identification.

The third app was the UK Ladybird Survey, which was developed by the University of Bristol and the Centre for Hydrology and Ecology. This is a single species app that uses a different process for identification and validation, because of the small number of ladybird species and the relatively distinctive look of the insect. Identification is made by the user who compares their photo with that of the 23 species images stored by the app. The data record is then submitted to project scientists who validate the record.

The researcher asked participants to complete a range of tasks using the apps, for instance, to make a 'sighting' and submit. Participants were also asked to explore other features of the apps to understand their role in supporting data collection functionality specifically the 'mission' and 'reputation' elements of Project Noah and iSpot respectively and whether they helped encourage participation. Testing sessions lasted approximately an hour and were followed by a focus group discussion.

The focus group discussion was audio-recorded. Recordings were transcribed and analysed using Thematic Analysis (Braun \& Clarke, 2006). Our themes included: image quality, screen visibility, connectivity and GPS, and manual entry of the location and landscape.

\section{Image Quality}

The photo is core to all these apps. Image quality therefore is paramount for identification purposes. However the Samsung handset did not take close-ups or ena- 
ble users to zoom. Although the study did not look at other models, it is likely that this will be an issue across most mobile phones. Users found this highly problematic, complaining of fuzzy images and the camera being too slow, i.e. the animal or insect had moved or the surface the insect was on moved, light levels were too low. As many of the subjects such as ladybirds are small, they require a close view for successful identification. The app itself did not enable zooming and this, forced users to work around the app in their attempt to identify the species. For example, in Figure 2 we can see a participant using the UK Ladybird Survey app, trying to identify the ladybird by referring to the actual ladybird, rather than the photo that he/she had just taken.

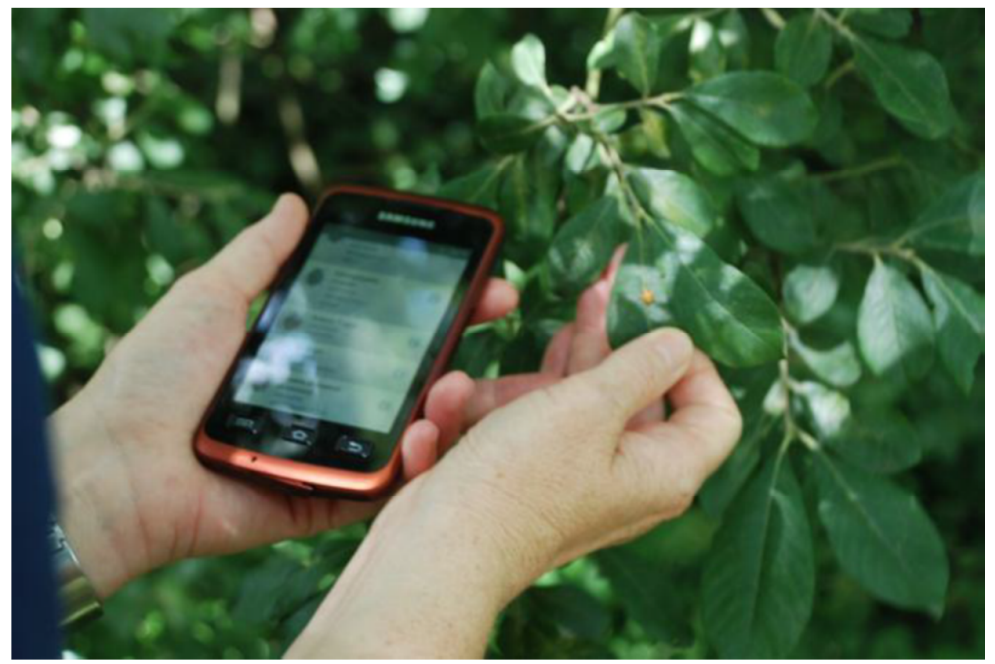

Figure 2. A participant referring to the ladybird, rather than the screen image, in order to identify it. This is because the screen image was poor and sunlight reflected on the screen reducing any ability to view the image

In our contextual observation of the Bioblitzes we had observed experts using SLR cameras mounted with $500 \mathrm{~mm}$ lens and using rapid continuous shutter functions in order to get the desired picture of their sightings, see Figure 3. Enabling users to achieve a similar functionality using a mobile phone app presents an important challenge: it is assumed that anyone with a smartphone can take photos and contribute data, however some images may not be of a good enough quality for species identification. 


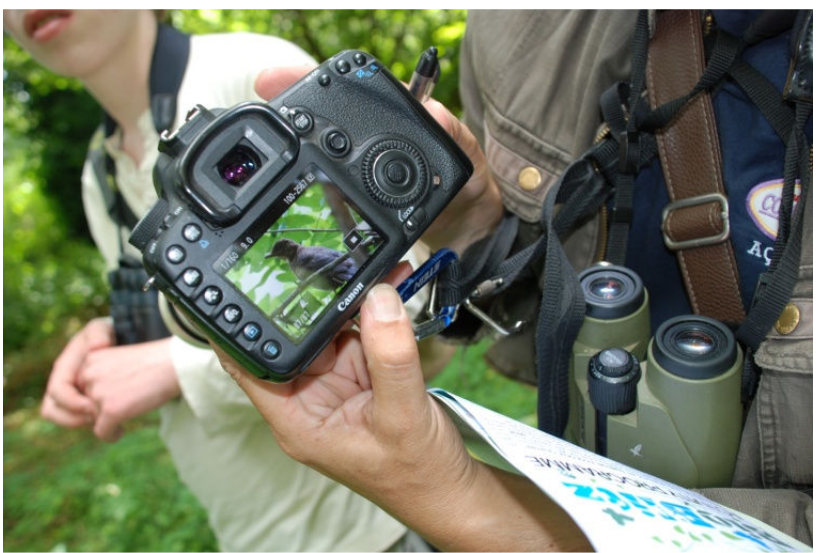

Figure 3. Viewing an image using a digital SLR camera during a Bioblitz. The camera was mounted with a $500 \mathrm{~mm}$ lens - this enabled the user to get a clear shot of the subject.

\section{Screen Visibility and Technology Use}

Direct sunlight hitting the screen reduced readability immensely and prevented effective use of the apps. This problem was exacerbated by interface designs that used small icons or required the user to switch through a number of small fields or tabs as part of the sequence of data entry, often leading to frustration. Rain was also a threat to data collection as mobile phones are not designed for the purpose of outdoor data collection. There was also ambiguity with the role of technology within data collection practices - while some participants clearly brought expensive equipment to the field (such as SLR), they seem reluctant to use smartphones or tablets in their field work. This might be due to the perception that the equipment is not robust enough or due to the context of use (as it is seen as interfering with the experience in the field).

\section{Connectivity and GPS}

Identifying an exact location for mobile devices depends on GPS signal. Waiting for a signal proved frustrating for many users who were faced with a revolving pre-loading icon for extended periods of time. This, with added absence of connectivity, meant sending a precise record was impossible.

App design does not help resolve these issues. If the Ladybird app specifies that finding a location will not take more than two minutes and the user is there for at least five, then the user is likely to find waiting frustrating. If the app requires 
the user to sign in at the last minute in order to send a record, as the UK Ladybird Survey does, and which in the absence of a connection appears to cause the app to fail then it is not surprising that the user becomes confused, frustrated and angry. For example, one participant complained:

"So everything's gone, lost. It's not right you do all your study and you put all the information in and you get stuck because of the ***application. It's a most frustrating thing!"

\section{Manual Entry of the Location and Landscape}

Location presents further complexity as the user is required to name the type of landscape. Participants described feeling uncertain whether they had entered the data correctly. For instance, is Hampstead Heath 'grassland' or a 'park'? What is 'heathland'? This information was, for instance, requested by iSpot.

Some participants could not understand why they needed to determine location with many suggesting that landscape/location functionality should be a background operation. In addition, some participants didn't understand why location needed to be part of the sequence of data entry at all, thereby misunderstanding an essential and fundamental requirement of ecological monitoring.

\section{Discussion and Conclusions}

Mobile sensing apps allow citizens to contribute to environmental monitoring research. However, as our studies revealed, there are several barriers to data collection. Technological factors, such as lack of connectivity, can prevent sample capture and lead to user frustration. Enabling storage and a 'send later' function (like WideNoise does) is one way to overcome this problem and can be significant in citizen science projects that require environmental monitoring; however this also places memory load on the user as they need to remember to send the record later. Enabling automatic submission when connected to a Wi-Fi network or $3 \mathrm{G} / 4 \mathrm{G}$ service is another possible solution; but limited in that accurate geo-localization data of the samples might not be available.

Environmental factors can also present barriers to data collection. Participants found it difficult to take noise samples on a busy street if the app involved too many steps. Weather conditions, such as the sun and rain, affected the screen visibility and the ease at which data could be collected.

Crucially, we argue that many of these insights were only possible because we conducted our research in the wild (Rogers, 2011). By testing the noise monitoring apps around the university campus, and testing the ecological monitoring apps in open heathland areas, we were able to uncover participants' experience of the apps 
within an intended context of use. Additionally, the 6-day diary study gave us insights into participants' experience of using (and not using) the apps within their day-to-day lives. Lifestyle and motivation factors, such as lack of time, routine, and forgetfulness, were identified as barriers for sustained contribution.

Conducting research in the wild is not an easy task. It can be difficult to recruit participants and to sustain their motivation over a long period of time. We found it helpful to recruit participants with a minimal/moderate interest in environmental issues. We also found it helpful to offer participants a small reimbursement for their time and to send diary participants regular reminders.

In future research it would be useful to investigate ways of sustaining citizens' participation in environmental monitoring activities. Based on our findings, we recommend emphasising how each person's contribution makes a difference and allowing participants to track their individual progress and the project's progress. Using the persuasive design literature as inspiration (Fogg, 2009), researchers could implementing different 'triggers' to prompt citizens to contribute, exploring which trigger participants like best. Furthermore, it would be useful to explore the impact of social motivations (Rotman et al., 2012). In our study of BioBlitzes we found that novices viewed it as a fun day out, where they could meet other people and learn more about nature. Similarly, we recommend that if environmental monitoring apps were designed to help citizens meet up and collect data in groups, and encouraged citizens to interact with each other, it is possible that social factors could play a strong role in sustaining participation.

Acknowledgments This research was funded by the EU FP7 projects Citizen Cyberlab (Grant agreement 317705) and the Everyaware project (Grant Agreement 265432). We would like to thank all of the participants that took part in our research studies.

\section{References}

Becker, M., Caminiti, S., Fiorella, D., Francis, L, Gravino, P., Haklay, M., Hotho, A., Loreto, V., Mueller, J., Ricchiuti, F., Servedio, V. D. P., Sirbu, A. and Tria, F. (2013). Awareness and Learning in Participatory Noise Sensing. PLOSone, December 2013. DOI: 10.1371/journal.pone.0081638

Braun, V. and Clarke, V. (2006). Using thematic analysis in psychology. Qualitative Research in Psychology, 3 (2), 77-101.

D’Hont, E., Stevens, M. and Jacobs, A. (2012). Participatory noise mapping works! An evaluation of participatory sensing as an alternative to standard techniques for environmental monitoring. Pervasive and Mobile Computing, 9 (5), 681-694.

European Commission. (1996). Green Paper on Future Noise. (COM(96) 540).

European Parliament and Council. (2002) Directive 2002/49/EC relating to the assessment and management of environmental noise. Official Journal of the European Communities, 12-26.

Fogg, B. J. (2009). A behavior model for persuasive design. Proceedings of the 4th International Conference on Persuasive Technology, article 40. 
Lane, N. D., Miluzzo, E., Lu, H., Peebles, D., Choudhury, T. and Campbell, A. T. (2010). A survey of mobile phone sensing. Communications Magazine, IEEE, 48(9), 140-150.

Rogers, Y. (2011). Interaction design gone wild: Striving for wild theory. Interactions, 18 (4), 58-62.

Rotman, D., Preece, J. and Hammock, J. (2012). Dynamic changes in motivation in collaborative citizen-science projects. Proceedings of the ACM 2012 conference on Computer Supported Cooperative Work, pp.217-227.

Stevens, M. (2012). Community memories for sustainable societies: The case of environmental noise. Doctoral dissertation. Vrije Universitetit Brussel.

\section{Apps}

iSpot. http://www.ispotnature.org/

NoiseWatch. http://eyeonearth.org/map/NoiseWatch/

Project Noah. http://www.projectnoah.org/

UK Ladybird Survey. http://www.ladybird-survey.org/

WideNoise. http://www.widetag.com/widenoise/ 